\title{
A Mathematical Model to Assess the Material Removal Rate during Gas-Assisted Electrical Discharge Drilling Process
}

\author{
Nishant Kumar Singh \\ Department of Mechanical Engineering, \\ Hindustan College of Science and Technology, Mathura, Uttar Pradesh, India. \\ Yashvir Singh \\ Department of Mechanical Engineering, \\ Graphic Era Deemed to be University, Dehradun, Uttarakhand, India. \\ Corresponding author: yashvirsingh21@gmail.com, yashvirsingh.me@geu.ac.in \\ Abhishek Sharma \\ Department of Mechanical Engineering, \\ G. L. Bajaj Institute of Technology and Management, Greater Noida, Uttar Pradesh, India.
}

(Received April 4, 2020; Accepted July 3, 2020)

\begin{abstract}
This research work investigates the use of Gas-Assisted Electrical Discharge Drilling (GAEDD) of high carbonchromium die steel. The poor material removal rate (MRR) is one of the profound drawbacks of the traditional Electrical Discharge Drilling (EDD) process. Hence, over the years researchers have been feeling the requisite to develop an advanced strategy that can enhance the MRR. This study has examined the utilization of compressed gas in conventional EDM under the constraint state to assess MRR. The impact of procedure parameters likedischarge current, pulse on time, duty cycle, electrode speed, and discharge gas pressure, on MRR, has been explored too. In addition, Variance Analysis (ANOVA) was performed to determine the significant parameters affecting the MRR. During the examination, a mathematical model was established MRR employing Buckingham $\pi$-theorem while the GAEDD was being applied. The experiment and anticipated values of the model show a noteworthy impact of the coefficient of thermal expansion in GAEDD of high carbon-chromium steel. In addition, the Response Surface Method (RSM) model has also been evolved. The comparative analysis of the models developed shows considerable agreement in anticipation. Moreover, the semiempirical model appears to be even more adaptable especially in comparison to the RSM-based model. In fact, the conclusion of this work is that the dimensional analysis model is an effective and reliable strategy to precise EDD response prediction.
\end{abstract}

Keywords- Electrical discharge maching, Drilling, Responses, Material removal rate, Response surface methodology.

\begin{tabular}{ll}
\multicolumn{2}{l}{ Nomenclature } \\
$\mathrm{I}_{\mathrm{p}}$ & Discharge Current \\
$\mathrm{T}_{\mathrm{on}}$ & Pulse-on Time \\
$\mathrm{DC}$ & Duty Cycle \\
$\mathrm{RPM}$ & Tool Rotation \\
$\mathrm{GP}$ & Gas Pressure \\
$\mathrm{K}$ & Thermal conductivity \\
$\sigma$ & Electrical conductivity \\
$\rho$ & Density \\
$\mathrm{C}_{\mathrm{p}}$ & Sp. Heat \\
$\theta$ & Melting point \\
$\mathrm{MRR}$ & Material removal rate
\end{tabular}


International Journal of Mathematical, Engineering and Management Sciences

Vol. 5, No. 6, 1333-1344, 2020

https://doi.org/10.33889/IJMEMS.2020.5.6.099

\section{Introduction}

Electrical discharge drilling (EDD) is a commonly adopted non-traditional method of machining that absorbs heat from a spark and excretes material from a rigid and stiff object, something that cannot be machined by a traditional technique. The technique is generally related to the production of die molds and aeronautical components (Ho and Newman, 2003). One of the real perplexities involved during EDD is the effective removal of eroded materials from electrodes gap. The phenomena of short circuit occurred when eroded material is collected between the electrodes gap. This leads to a reduced rate of material removal (MRR) and poor surface integrity (Singh et al., 2016, 2017).

So as to enhance the effectiveness of the EDM procedure, scientists look into different techniques regarding a few customary, innovative algorithm and creative frameworks. Due to the uncertainty and arbitrary nature of the different procedures and multiple methodological variables, the ideal approach has yet to be developed by researchers. Tsai and Wang (2001) applied Buckingham's pihypothesis to measures the SR during the EDM operation. In addition to the machining parameters, the electrical and non-electrical parameters used to create the model are also taken into account. The anticipated value of SR through model has a good agreement with the value obtained through the experiment. Yahya and Manning (2004) analyzed the significant factors affecting the EDM operation. They notable parameters were ascertained by using the ANOVA and these parameters were considered to evolve a model to predict the MRR during the EDM process. Kumar and Khamba (2009) proposed $\mu$-model to measure the MRR using the dimensional technique during the ultrasonic-assisted EDM of titanium alloys specimens. Patil and Brahmankar (2010) developed a mathematical model based on Buckingham's $\pi$-theorem to assess the MRR during the wire-EDM (WEDM) process. The average error between actual and predicted values was found to be less than $5 \%$. Yahya et al. (2012) performed comparative studies of finding obtained through the predictive model based on Buckingham's $\pi$-theorem and Artificial Neural Network (ANN) for the EDM operation. Dave et al. (2013) proposed the model using the dimensional analysis method to anticipate the MRR during the orbital-assisted EDM. The root means square error between actual and predicted values was found to be 3\% (approx). Kumar et al. (2014) applied statistical techniques to analyze the impacts of process factors during WEDM on notable process output such as MRR and over-cut. In the course of the investigation, they created a predictive model centered on $\pi$-theorem of Buckingham to assess the process output. Bobbili et al. (2015) performed experimental analysis and proposed mathematical model to measures the WEDM responses like MRR and SR. Finding suggested the there is a good accord between measures and predicted values by developed model. In the course of an investigation, Bhaumik et al. (2018) developed a semiempirical technique to assess MRR and radial overcut (ROC) during EDM with tungsten carbide electrode. Apart from the machining parameters, work material properties are also considered for the development of a predictive model using Buckingham's $\pi$-theorem. Kumar et al. (2015) proposed a mathematical model for the assessment of tool wear during powder mixed EDM using Buckingham's $\pi$-theorem. Estimated results from the mathematical model were verified and observed to be in agreement with the experimental outcome. Phate and Toney (2019) studied WEDM process responses by applying dimensional analysis and artificial neural network (ANN) techniques. It was evident from the coefficient of determination that both dimensional analysis and ANN techniques are qualified to estimate SR and MRR. In addition, the models developed using the ANN methodology have been discovered to be somewhat more accurate than the dimensional analysis techniques. Recently, Singh et al. (2016, 2017) proposed a semi-empirical model to measure the surface roughness during the argon-based EDM operation. Finding, reveal that the average error between experimental and predicted values are less than 3\%. Further, they discover 
that the semi-empirical model projected the EDM output quite accurately in comparison of regression model.

On the basis of the study of literature available, it tends to be seen that the greater part of the exploration work concentrated on either conventional or wire-EDM. Singh et al. $(2016,2017)$ earlier trial examinations have discovered the benefits of gas-assisted EDM when contrasted with rotational EDM regarding MRR and EWR. From a review of previous literature, no investigation of a statistical model could be identified, in perspective on thermal as well as mechanical properties, which would make certain improvement in MRR during gas based EDM. The model based on a dimensional analysis, previously developed, was largely dependent on the parameters of the machining operation and various properties of the workpiece. The advancement of the model taking into account dielectric characteristics, including compressed gas supply and electrode revolution speed for additional studies, has not been documented to the author 's information. From a review of past research work, it was additionally seen that no work is accounted for on parts of material removal mechanism in inert gas-based EDM operation.

In light of the above mentioned problems, the purpose of the contemporary investigation is to explore and establish a mathematical model for predicting MRR by introducing high-pressure argon gas via perforated electrode in conventional EDD.

\section{Materials and Methods}

\subsection{Workpiece and Electrode Material}

The experiments have been performed on carbon-chromium steel samples, employing rounded copper as tool. The sample utilized has a rectangular dimension $(15 \times 10 \times 10 \mathrm{~mm})$ and having wear resistance of 55HRC. Table 1 reveals the specified specimen's chemical composition.

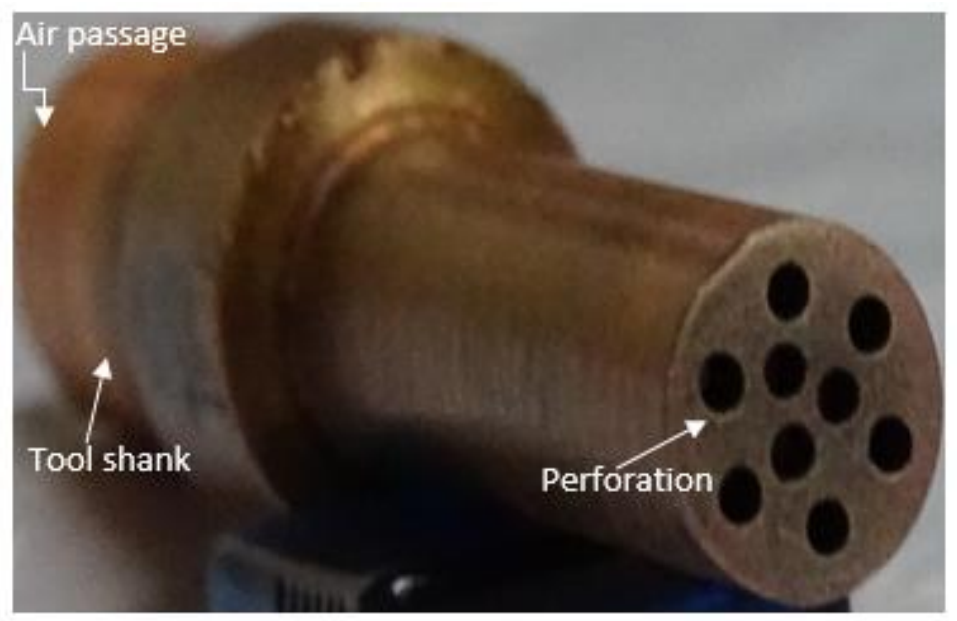

Figure 1. Image of the tool

Table 1. Chemical composition of the workpiece

\begin{tabular}{|c|c|c|c|c|c|c|}
\hline $\mathbf{C r}$ & $\mathbf{C}$ & $\mathbf{S i}$ & $\mathbf{M n}$ & $\mathbf{P}$ & $\mathbf{S}$ & $\mathbf{F e}$ \\
\hline 11.05 & 2.25 & 0.50 & 0.25 & 0.09 & 0.05 & Rest. \\
\hline
\end{tabular}


International Journal of Mathematical, Engineering and Management Sciences

Vol. 5, No. 6, 1333-1344, 2020

https://doi.org/10.33889/IJMEMS.2020.5.6.099

\subsection{Experimental Procedure}

The machining execution period for argon-assisted EDM with a perforated tool has been set to 15 minutes for each and every experiment. The EDM oil-gas mixed dielectric was used in GAEDD process. During machining, the electrode was immersed in the dielectric fluid. The argon gas was provided directly through the perforated electrode in to the electrodes gap. The gas pressure was modified to prevent the formation of a completely dry or wet state. Five variable factors, like those of discharge current, pulse duration, duty cycle, electrode rpm, and gas pressure, were utilized to conduct the experiment. The range of selected control factors was decided on the grounds of preliminaries experiments and machine limit. For conventional EDM, high-pressure argon gas was being used to avoid oxidation reactions, the risk of flame and hazards throughout machining operations. Table 2 displays the variety of machining factors used for the current study. Schematic of tool electrode is depicted in Figure 1. The proposed work plans experiments with Central Composite Rotatable Design (CCRD) way to assess effects of various process variables on output. A total of 32 experiments with independent variables at 5 different levels were performed using CCRD approach. Each experiment was conducted thrice to minimize experimental errors, and its mean value was taken as the response to the process. For each experiment in the GAEDD method the evaluated MRR values were summarized in Table 3.

Table 2. Control factors with their levels

\begin{tabular}{|l|c|c|c|c|c|}
\hline \multicolumn{1}{|c|}{ Factors } & \multicolumn{5}{c|}{ Levels } \\
\cline { 2 - 6 } & $\mathbf{- 2}$ & $\mathbf{- 1}$ & $\mathbf{0}$ & $\mathbf{1}$ & $\mathbf{2}$ \\
\hline Discharge Current $\left(\mathrm{I}_{\mathrm{p}}\right)(\mathrm{A})$ & 3 & 4 & 5 & 6 & 7 \\
\hline Pulse-on Time $\left(\mathrm{T}_{\text {on }}\right)(\mu \mathrm{s})$ & 100 & 200 & 300 & 400 & 500 \\
\hline Duty Cycle $(\mathrm{DC})$ & 0.52 & 0.58 & 0.64 & 0.70 & 0.76 \\
\hline Tool Rotation $(\mathrm{rpm})$ & 100 & 300 & 500 & 700 & 900 \\
\hline Gas Pressure $(\mathrm{GP})(\mathrm{mm} \mathrm{Hg})$ & 3 & 6 & 9 & 12 & 15 \\
\hline
\end{tabular}

The MRR was determined as an extent of wear volume of a workpiece to the machining time (Srivastava and Pandey, 2013).

$\operatorname{MRR}\left(\mathrm{mm}^{3} / \mathrm{min}\right)=\frac{\text { Wear volume of the specimen }}{\text { Machining time }}$

Machined workpiece have been cleaned in acetone. The electronic gauging balance was used for the calculation of weight. Electronic clock (precision of 0.1 seconds) was used to ensure precise machining time figuring.

Table 3. Measured responses corresponding to each trial of GAEDD process

\begin{tabular}{|c|c|c|c|c|c|c|}
\hline \multirow{2}{*}{ Run Order } & $\begin{array}{c}\text { Discharge } \\
\text { Current }\end{array}$ & $\begin{array}{c}\text { Pulse-on } \\
\text { Time }\end{array}$ & Duty Cycle & Tool Speed & $\begin{array}{c}\text { Gas } \\
\text { Pressure }\end{array}$ & MRR \\
\cline { 2 - 7 } & $(\mathrm{A})$ & $(\mu \mathrm{s})$ & & $(\mathrm{rpm})$ & $(\mathrm{mm} \mathrm{Hg})$ & $(\mathrm{mg} / \mathrm{min})$ \\
\hline 1 & 4 & 200 & 0.58 & 300 & 12 & 8.70 \\
\hline 2 & 6 & 200 & 0.58 & 300 & 6 & 13.19 \\
\hline 3 & 4 & 400 & 0.58 & 300 & 6 & 7.25 \\
\hline 4 & 6 & 400 & 0.58 & 300 & 6 & 13.37 \\
\hline 5 & 4 & 200 & 0.7 & 300 & 6.94 \\
\hline 6 & 6 & 200 & 0.7 & 300 & 12 & 17.75 \\
\hline
\end{tabular}


International Journal of Mathematical, Engineering and Management Sciences

Vol. 5, No. 6, 1333-1344, 2020

https://doi.org/10.33889/IJMEMS.2020.5.6.099

Table 3 continuied...

\begin{tabular}{|c|c|c|c|c|c|c|}
\hline 7 & 4 & 400 & 0.7 & 300 & 12 & 8.65 \\
\hline 8 & 6 & 400 & 0.7 & 300 & 6 & 12.65 \\
\hline 9 & 4 & 200 & 0.58 & 700 & 6 & 6.98 \\
\hline 10 & 6 & 200 & 0.58 & 700 & 12 & 16.15 \\
\hline 11 & 4 & 400 & 0.58 & 700 & 12 & 9.42 \\
\hline 12 & 6 & 400 & 0.58 & 700 & 6 & 10.35 \\
\hline 13 & 4 & 200 & 0.7 & 700 & 12 & 10.69 \\
\hline 14 & 6 & 200 & 0.7 & 700 & 6 & 15.93 \\
\hline 15 & 4 & 400 & 0.7 & 700 & 6 & 7.65 \\
\hline 16 & 6 & 400 & 0.7 & 700 & 12 & 5.85 \\
\hline 17 & 3 & 300 & 0.64 & 500 & 9 & 5.54 \\
\hline 18 & 7 & 300 & 0.64 & 500 & 9 & 21.42 \\
\hline 19 & 5 & 100 & 0.64 & 500 & 9 & 15.21 \\
\hline 20 & 5 & 500 & 0.64 & 500 & 9 & 6.43 \\
\hline 21 & 5 & 300 & 0.52 & 500 & 9 & 8.25 \\
\hline 22 & 5 & 300 & 0.76 & 500 & 9 & 12.35 \\
\hline 23 & 5 & 300 & 0.64 & 100 & 9 & 9.13 \\
\hline 24 & 5 & 300 & 0.64 & 900 & 9 & 7.67 \\
\hline 25 & 5 & 300 & 0.64 & 500 & 3 & 7.78 \\
\hline 26 & 5 & 300 & 0.64 & 500 & 15 & 10.95 \\
\hline 27 & 5 & 300 & 0.64 & 500 & 9 & 10.78 \\
\hline 28 & 5 & 300 & 0.64 & 500 & 9 & 10.98 \\
\hline 30 & 5 & 300 & 0.64 & 500 & 9 & 10.69 \\
\hline 31 & 5 & 300 & 0.64 & 500 & 9 & 10.67 \\
\hline 32 & 5 & 300 & 0.64 & 500 & 9 & 10.63 \\
\hline
\end{tabular}

\subsection{Analysis of Variance (ANOVA)}

ANOVA was performed to know the notable factors influencing MRR during the GAEDD. The ANOVA model of MRR was depicted in Table 4. The measurements of 'Prob > F' were found to be less than 0.05 (95 percent confidence) for the ANOVA model. Therefore, the conclusion may be drawn that the variables in the model had a major impact on the EDD output. The statistical model for MRR in the form of equations 2 had shown as below.

$$
\begin{aligned}
& M R R=5.30-\left(3.73 \times I_{p}\right)-\left(0.0216 \times T_{o n}\right)+(10.9 \times D C)+(0.0131 \times R P M)+(0.032 \times \\
& G P)+\left(0.712 \times I^{2}{ }_{p}\right)-\left(0.000015 \times R P M^{2}\right)
\end{aligned}
$$

\begin{tabular}{|c|c|c|c|c|c|c|c|}
\hline Source & $D F$ & SS & $M S$ & $F$ & $P$ & $R^{2}$ & \\
\hline Regression & 8 & 432.15 & 54.01 & 62.74 & 0 & 0.95 & \multirow{6}{*}{$\begin{array}{l}F_{(0.05,8,23) ;}^{\text {standard }}=2.80 \\
F^{\text {regression }}>F_{(0.05,8,23)}^{\text {standard }} \\
F_{(0.05,8,23) ;}^{\text {standaed }}=1.76 \\
F^{\text {lack-of }- \text { fit }}<F_{(0.05,18,23)}^{\text {standard }} \text { The method is suitable and } \\
\text { the lack of fit is negligible. }\end{array}$} \\
\hline Linear & 5 & 405.35 & & & & & \\
\hline Square & 2 & 20.72 & & & & & \\
\hline Interaction & 1 & 6.07 & & & & & \\
\hline Residual error & 23 & 19.80 & 0.86 & & & & \\
\hline Lack-of-Fit & 18 & 16.77 & & 1.54 & 0.335 & & \\
\hline
\end{tabular}

Table 4. ANOVA table for MRR 
International Journal of Mathematical, Engineering and Management Sciences

Vol. 5, No. 6, 1333-1344, 2020

https://doi.org/10.33889/IJMEMS.2020.5.6.099

\subsection{Dimensional Analysis for Assessing MRR During GAEDD}

The dimensional investigation is a strategy applied to acquire a specific arrangement of data about a considered occurrence. Absolute preparation of dimensionless variables can be obtained through dimensional analysis. The methodology of dimensional technique is primarily always had to mitigate the uncertain intricacies of physical problems by minimizing the number of variables that don't have a fundamental effect on the particular problem (Sonin, 2001). In the event that the problem is assumed to have ' $n$ ' variables and in the situation that ' $k$ ' implies elemental dimensions, then the dimensional perusal minimizes the difficulty to just $\pi$-dimensionless terms. For the most part, ' $n-k$ ' refers to the total dimensionless terms that manage the issue. The attributes of physical quantities are shown in Table 5.

Table 5. Various attributes of physical quantities

\begin{tabular}{|l|l|l|l|}
\hline \multirow{5}{*}{ Process Factors } & Parameters & value & Dimensions \\
\cline { 2 - 4 } & Current & & $Q T^{-1}$ \\
\cline { 2 - 4 } & Pulse-duration & & $T$ \\
\cline { 2 - 4 } & Tool speed & & $T^{-1}$ \\
\cline { 2 - 4 } & Gas pressure & & $M L^{-1} T^{-2}$ \\
\cline { 2 - 4 } & Duty cycle & $50 \mathrm{~W} / \mathrm{m}-\mathrm{K}$ & 1 \\
\hline \multirow{5}{*}{ Material Properties } & Thermal conductivity & $0.01824 \mathrm{~S} / \mathrm{m}$ & $M L T^{-3} \theta^{-1}$ \\
\cline { 2 - 4 } & Electrical conductivity & $7700 \mathrm{Kg} / \mathrm{m}^{3}$ & $\mathrm{M}^{-1} \mathrm{~L}^{-3} T Q^{2}$ \\
\cline { 2 - 4 } & Density & $0.46 \mathrm{Cal} / \mathrm{S} \mathrm{mole}{ }^{\circ} \mathrm{C}$ & $\mathrm{ML}^{-3}$ \\
\cline { 2 - 4 } & Sp. Heat & $1421 \mathrm{C}^{\mathrm{C}}$ & $L^{2} T^{-2} \theta^{-1}$ \\
\cline { 2 - 4 } & Melting point & & $\theta$ \\
\hline Response & Material removal rate & $L^{3} T^{-1}$ \\
\hline
\end{tabular}

Function:

$M R R=f\left(I_{p}, T_{o n}, R P M, G P, D C, K, \sigma, \rho, C_{p}, \theta\right)$

In this scenario, the matrix is five, so there are 11 factors. Consequently, as per Buckingham postulate, there are six $\pi$--terms and there is an equation for the for the given problem is:

$$
\begin{aligned}
& {\left[L^{3} T^{-1}\right]^{k_{1}}\left[Q T^{-1}\right]^{k_{2}}[T]^{k_{3}}\left[T^{-1}\right]^{k_{4}}\left[M L^{-1} T^{-2}\right]^{k_{5}}\left[M L T^{-3} \theta^{-1}\right]^{k_{7}}} \\
& {\left[M^{-1} L^{-3} T Q^{2}\right]^{k_{8}}\left[M L^{-3}\right]^{k_{9}}\left[L^{2} T^{2} \theta^{-1}\right]^{k_{10}}[\theta]^{k_{11}}=\left[M^{0} L^{0} T^{0} Q^{0} \theta^{0}\right]} \\
& M R R=(0.0007138) \times Z\left(I_{p} \rho C_{p}^{1.5} / K^{1.5} \sigma^{0.5}\right)^{\alpha} \times\left(T_{\text {on }} \rho C_{p}{ }^{2} \theta / K\right)^{\beta} \times \\
& \left(R P M K / \rho C_{p}{ }^{2} \theta\right)^{\gamma} \times\left(G P / \rho C_{p} \theta\right)^{\delta} \times(D C)^{\lambda}
\end{aligned}
$$

where, the constant is $\mathrm{Z}$ and the undefined exponents are $\alpha, \beta, \gamma, \delta$ and $\lambda$. The non-linear method is applied to evaluate the constant and unidentified exponents. Estimates of $Z, \alpha, \beta, \gamma, \delta$ and $\lambda$ are shown to be $281,729169.70,1.0913,-0.4182,-0.0125,0.2907$ and 0.9505 separately. 
The point by point procedure is altogether clarified in Appendix A. Condition (5) can be written in the accompanying structure:

$$
\begin{aligned}
& M R R=(212.12) \times\left(I_{p} \rho C_{p}^{1.5} / K^{1.5} \sigma^{0.5}\right)^{1.0913} \times\left(T_{\text {on }} \rho C_{p}{ }^{2} \theta / K\right)^{-0.4182} \times \\
& \left(R P M K / \rho C_{p}{ }^{2} \theta\right)^{-0.0125} \times\left(G P / \rho C_{p} \theta\right)^{0.2907} \times(D C)^{0.9505}
\end{aligned}
$$

The competency of the created model has been verified by the mean error (ME), root means square error (RMSE) and average percentage error ( $\Delta \mathrm{E} \%$ ) (Singh et al., 2019). The accompanying conditions were utilized for these investigations:

$$
\begin{aligned}
& M E=\frac{1}{N} \sum_{i=1}^{N}\left(A_{i}-A\right) \\
& R M S E=\sqrt{\frac{1}{N}} \sum\left(A_{i=1}^{N}-A\right)^{2} \\
& \Delta E(\%)=\frac{1}{N} \sum\left(A_{i=1}^{N}-A\right)
\end{aligned}
$$

Here, $N$ is the entire collection of data, $A_{i}$ is the computation of the expected information, and $A$ is the measure anticipated by the model on the basis of the dimensional analysis. The reliability of the perception model was evaluated utilizing the ME, RMSE, $(\Delta \mathrm{E} \%)$ and is recorded in Table 6. From such attributes, it appears to be presumed that the model produced succesively empowers to correct and realistic expectations.

Table 6. Accuracy of predictive model

\begin{tabular}{|c|c|c|c|}
\hline Process output & ME & RMSE & ( 1 E\%) \\
\hline MRR & 0.028 & 0.752 & 2.80 \\
\hline
\end{tabular}

Table 7. Affirmation of the MRR prediction through the proposed model

\begin{tabular}{|c|c|c|c|c|c|c|c|}
\hline \multirow{2}{*}{ Exp. No. } & \multicolumn{6}{|c|}{ Machining parameters } & \multicolumn{2}{c|}{ MRR $\left(\mathbf{m m}^{\mathbf{3}} / \mathbf{m i n}^{\prime}\right.$} \\
\cline { 2 - 8 } & $I_{p}$ & $T_{\text {on }}$ & $D C$ & $r p m$ & $G P$ & Predict. & Exp. \\
\hline 1 & 7 & 300 & 0.58 & 500 & 15 & 20.03 & 19.66 \\
\hline 2 & 4 & 100 & 0.64 & 300 & 9 & 10.81 & 10.76 \\
\hline 3 & 5 & 200 & 0.76 & 100 & 6 & 10.75 & 10.55 \\
\hline
\end{tabular}

Examinations were led under various parametric combinations to approve the developed model. The forecasting capability of any model can be resolved uniquely with an altogether new 
arrangement of data. Consequently, the created model was tried utilizing data, not recently utilized during the experimentation procedure. Subtleties are displayed in Table 7. It very well may be gathered from Table 7 that the developed model can foresee MRR during the GAEDD procedure with great precision.

\section{Results and Discussion}

The experiment and the anticipated measures taken through the semi-empirical model were discussed in the following subsections.

\subsection{Process Factors Effect on MRR During GAEDD}

The comparison of regression, semiempirical and observational outcomes is shown in Figure 2(a), Figure 2(b), Figure 2(c) and Figure 2(d). The outcomes demonstrate a decent understanding between test esteems and anticipated qualities in the semi-experimental model. It concludes from these figures that the semi-empirical approach actually results in valid and accurate results in the regression model correlation. Semi-empirical models, which had developed earlier, were largely dependent on the machining operation variables, electrical, physical and thermal attributes of the workpiece. Besides the above-mentioned properties, the dielectric attributes, the discharge gas pressure and the electrode revolution were also taken into account in the current analysis. In addition, this model can be related to look at MRR just as specific procedure responses (Table 8).

The exploratory and anticipated measures of MRR during the GAEDD procedure are displayed in Figure 3. The results of the tests and model anticipation measures of the MRR during the GAEDD procedure can be considered as per agreement.

The normal error between the anticipated and observation values is around 5\%. The commitment level of each parameter and the response error, determined by the calculation of the error variance $(V e)$ and the sum of the regression squares as acquired from the ANOVA table. (Srivastava and Pandey, 2013), can be defined as pursues:

$$
\Delta Y=t \alpha / 2, D F \sqrt{V_{e}}
$$

where $\Delta \mathrm{Y}$ represents the error accordingly, the dimension of certainty interim is meant by $\alpha$ and is considered as 0.05 . The difference of error of expected procedure response is indicated by $V e$. The determined fallacy can be in Figure 3 by the use of error bars. 
International Journal of Mathematical, Engineering and Management Sciences

Vol. 5, No. 6, 1333-1344, 2020

https://doi.org/10.33889/IJMEMS.2020.5.6.099

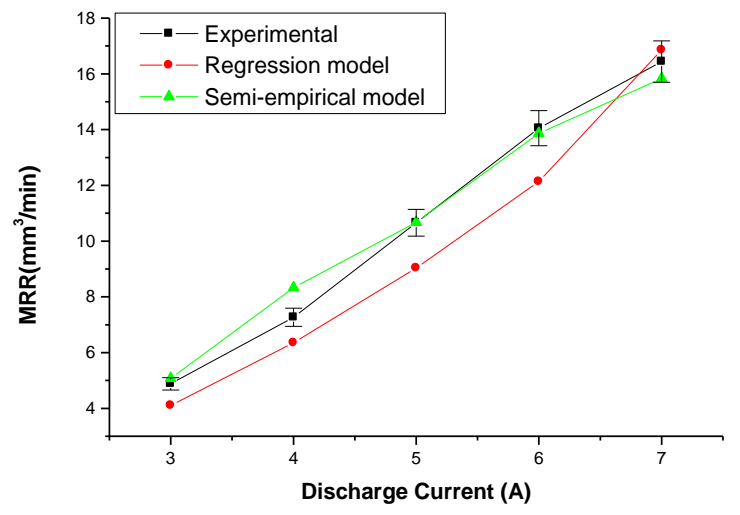

(a)

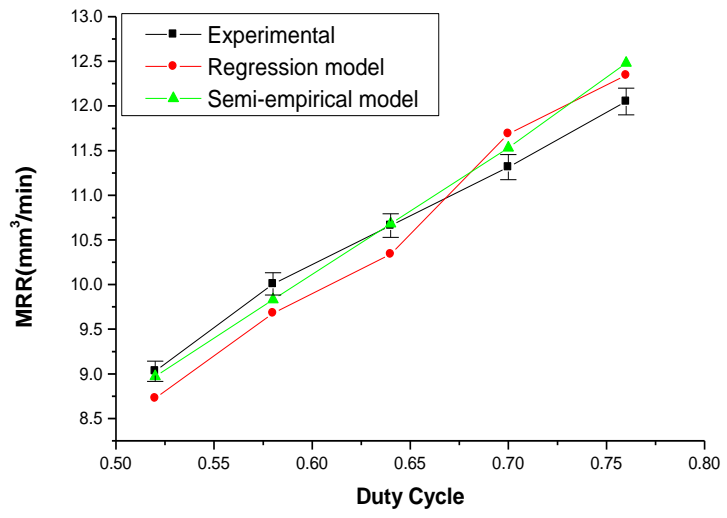

(c)

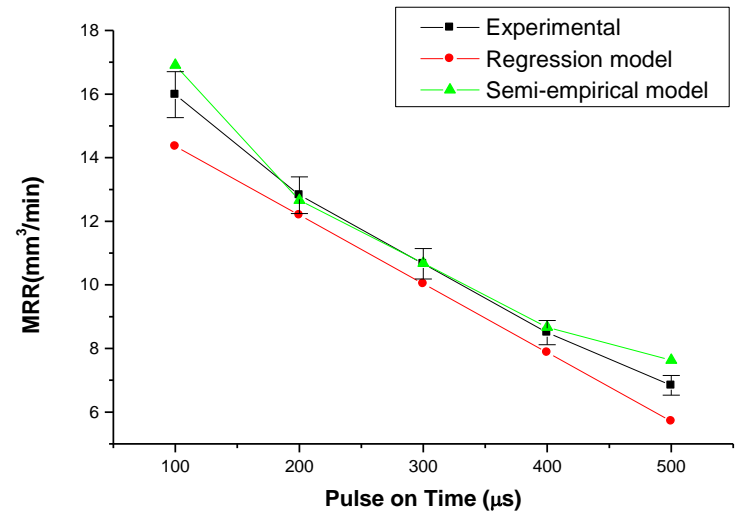

(b)

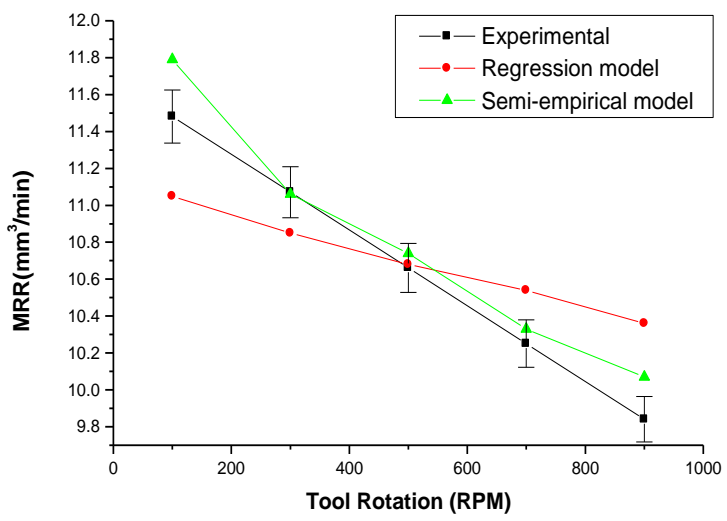

(d)

Figure 2. The comparative study of MRR between the experimental, regression and semi-empirical models for (a) current (b) pulse duration (c) duty cycle (d) tool rotation

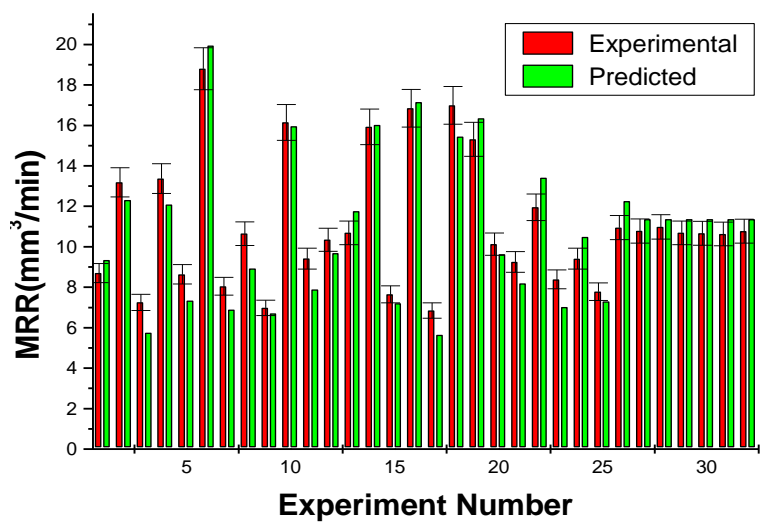

Figure 3. A comparative studies of experimental data and MRR model estimates during the GAEDD process 
International Journal of Mathematical, Engineering and Management Sciences

Vol. 5, No. 6, 1333-1344, 2020

https://doi.org/10.33889/IJMEMS.2020.5.6.099

Table 8 . The error between the anticipated and the exploratory outcomes

\begin{tabular}{|c|c|c|c|}
\hline Run order & Experimental (MRR) & Predicted (MRR) & Error \\
\hline 1 & 8.70 & 9.34 & -0.64 \\
\hline 2 & 13.19 & 12.31 & 0.88 \\
\hline 3 & 7.25 & 5.75 & 1.5 \\
\hline 4 & 13.37 & 12.08 & 1.29 \\
\hline 5 & 8.64 & 7.33 & 1.31 \\
\hline 6 & 18.80 & 19.95 & -1.15 \\
\hline 7 & 8.05 & 6.89 & 1.16 \\
\hline 8 & 10.65 & 8.72 & 1.93 \\
\hline 9 & 6.98 & 6.71 & 0.27 \\
\hline 10 & 16.15 & 15.96 & 0.19 \\
\hline 11 & 9.42 & 7.78 & 1.64 \\
\hline 12 & 10.35 & 9.69 & 0.66 \\
\hline 13 & 10.69 & 11.76 & -1.07 \\
\hline 14 & 15.93 & 16.02 & -0.09 \\
\hline 15 & 7.65 & 7.21 & 0.44 \\
\hline 16 & 16.85 & 17.15 & -0.3 \\
\hline 17 & 6.85 & 5.64 & 1.21 \\
\hline 18 & 16.99 & 15.45 & 1.54 \\
\hline 19 & 15.31 & 16.35 & -1.04 \\
\hline 20 & 10.13 & 9.63 & 0.5 \\
\hline 21 & 9.25 & 8.19 & 1.06 \\
\hline 22 & 11.95 & 13.91 & -1.96 \\
\hline 23 & 8.39 & 6.91 & 1.48 \\
\hline 24 & 9.41 & 10.68 & -1.27 \\
\hline 25 & 7.78 & 7.30 & 0.48 \\
\hline 26 & 10.95 & 12.66 & -1.71 \\
\hline 27 & 10.78 & 11.36 & -0.58 \\
\hline 28 & 10.98 & 11.36 & -0.38 \\
\hline 29 & 10.69 & 11.36 & -0.67 \\
\hline 30 & 10.67 & 11.36 & -0.69 \\
\hline 31 & 10.63 & 11.36 & -0.73 \\
\hline 32 & 10.77 & 11.36 & -0.59 \\
\hline
\end{tabular}

\section{Conclusions}

EDD of high carbon-chromium steel was successfully performed in this work by a gas-assisted perforated electrode.

- ANOVA was carried out in order to learn the influence of different control factors on MRR. The far more influential parameter controlling the MRR, supplemented by pulse time and gas pressure, was observed to be the discharge current.

- A dimensional analysis based MRR model was developed based on the predominant machining parameters and thermo-physical attributes of the workpiece. The exploratory and expected outcomes of the MRR during the GAEDD method were ascertained to be as per the agreement. The relative deviation between the expected and exploratory outputs was less than $3 \%$.

- Corroborative assessments have been carried out in order to reaffirm the established model.This appears to benefit from the finding that the develop model can measure MRR with great accuracy during the GAEDD process.

- A correlation has been formed between the regression and dimensional analysis based models to differentiate the most reliable between them. The dimensional analysis model was shown to anticipate process output most precisely when compared with the regression model. 
International Journal of Mathematical, Engineering and Management Sciences

Vol. 5, No. 6, 1333-1344, 2020

https://doi.org/10.33889/IJMEMS.2020.5.6.099

- This study can be extended to the development of semi-empirical models for electrode wear rate, surface finish and surface crack length employing Buckingham's $\pi$-theorem. Empirical model can be employed using a RSM together with a multiple regression method.

Conflict of Interest

There are no potential conflicts here anyway.

\section{Acknowledgement}

This research was a combined effort, and also was reasonably endorsed under the HCST/2017/018 fund by the Hindustan College of Science and Technology (India).

\section{References}

Bhaumik, S., Maggirwar, R., Datta, S., \& Pathak, S.D. (2018). Analyses of anti-wear and extreme pressure properties of castor oil with zinc oxide nano friction modifiers. Applied Surface Science, 449, 277-286.

Bobbili, R., Madhu, V., \& Gogia, A.K. (2015). Modelling and analysis of material removal rate and surface roughness in wire-cut EDM of armour materials. Engineering Science and Technology, an International Journal, 18(4), 664-668.

Dave, H.K., Desai, K.P., \& Raval, H.K. (2013). Development of semi empirical model for predicting material removal rate during orbital electro discharge machining of Inconel 718. International Journal of Machining and Machinability of Materials, 13(2-3), 215-230.

Ho, K.H., \& Newman, S.T. (2003). State of the art electrical discharge machining (EDM). International Journal of Machine Tools and Manufacture, 43(13), 1287-1300.

Kumar, A., Kumar, V., \& Kumar, J. (2014). Semi-empirical model on MRR and overcut in WEDM process of pure titanium using multi-objective desirability approach. Journal of the Brazilian Society of Mechanical Sciences and Engineering, 37(2), 689-721.

Kumar, J., \& Khamba, J.S. (2009). Modeling the material removal rate in ultrasonic machining of titanium using dimensional analysis. The International Journal of Advanced Manufacturing Technology, 48(14), 103-119.

Kumar, S., Singh, R., Batish, A., \& Singh, T.P. (2015). Modeling the tool wear rate in powder mixed electrodischarge machining of titanium alloys using dimensional analysis of cryogenically treated electrodes and workpiece. Proceedings of the Institution of Mechanical Engineers, Part E: Journal of Process Mechanical Engineering, 231(2), 271-282.

Patil, N.G., \& Brahmankar, P.K. (2010). Determination of material removal rate in wire electro-discharge machining of metal matrix composites using dimensional analysis. The International Journal of Advanced Manufacturing Technology, 51(5-8), 599-610.

Phate, M.R., \& Toney, S.B. (2019). Modeling and prediction of WEDM performance parameters for Al/SiCp MMC using dimensional analysis and artificial neural network. Engineering Science and Technology, an International Journal, 22(2), 468-476.

Singh, N.K., Pandey, P. M., \& Singh, K.K. (2017). Experimental investigations into the performance of EDM using argon gas-assisted perforated electrodes. Materials and Manufacturing Processes, 32(9), 940-951.

Singh, N.K., Pandey, P.M., \& Singh, K.K. (2016). EDM with an air-assisted multi-hole rotating tool. Materials and Manufacturing Processes, 31(14), 1872-1878. 
International Journal of Mathematical, Engineering and Management Sciences

Vol. 5, No. 6, 1333-1344, 2020

https://doi.org/10.33889/IJMEMS.2020.5.6.099

Singh, N.K., Pandey, P.M., Singh, K.K., \& Sharma, M.K. (2016). Steps towards green manufacturing through EDM process: a review. Cogent Engineering, 3(1), 1272662.

Sonin, A.A. (2001). Dimensional analysis: Technical report, Massachusetts Institute of Technology.

Srivastava, V., \& Pandey, P.M. (2013). Experimental investigation on electrical discharge machining process with ultrasonic-assisted cryogenically cooled electrode. Proceedings of the Institution of Mechanical Engineers, Part B: Journal of Engineering Manufacture, 227(2), 301-314.

Tsai, K.M., \& Wang, P.J. (2001). Semi-empirical model of surface finish on electrical discharge machining. International Journal of Machine Tools and Manufacture, 41(10), 1455-1477.

Yahya, A., \& Manning, C.D. (2004). Determination of material removal rate of an electro-discharge machine using dimensional analysis. Journal of Physics D: Applied Physics, 37(10), 1467.

Yahya, A., Trias, A., Erawan, M.A., Nor Hisham, K., Khalil, K., \& Rahim, M.A.A. (2012). Comparison studies of electrical discharge machining (EDM) process model for low gap current. Advanced Materials Research, 433-440, 650-654. 